\title{
High-density impulse noise removal using fuzzy mathematical morphology
}

\author{
M. González-Hidalgo ${ }^{1}$ S. Massanet ${ }^{1}$ A. Mir $^{1}$ D. Ruiz-Aguilera ${ }^{1}$ \\ ${ }^{1}$ Dept. of Math. and Comp. Science, University of the Balearic Islands, 07122 Palma, Spain
}

\begin{abstract}
This paper proposes a filtering method for highdensity impulse noise removal based on the fuzzy mathematical morphology using t-norms. The method is a two phased method. In the first phase, an impulse noise detector based on the fuzzy tophat transforms is used to identify pixels which are likely to be contaminated by noise. In the second phase, the image is restored using a specialized regularization method using fuzzy open-close or fuzzy close-open sequences applied only to those selected contaminated pixels and applying then a block smart erase algorithm. Experimental results show that the proposed algorithm presents a better performance in terms of edge preservation and noise suppression than other nonlinear filtering methods, including the presented in [1], in which this method is based on.
\end{abstract}

Keywords: Mathematical morphology, t-norm, residual implication, high probability impulse noise, noise reduction, nonlinear filter, open-close filter.

\section{Introduction}

Digital images are systematically affected by noise during their acquisition, transmission or recording. This is a major problem for many image processing techniques since they cannot work well in a noisy environment. Consequently, a preprocessing step to deal with this fact is mandatory. Thus, in artificial vision, many techniques of interpretation, measurement, segmentation or detection of structures require the removal, reduction and smoothing of noise in order to improve their performance. However, the noise removal techniques must be applied looking for a compromise between the effective suppression of the noise while preserving the fine texture and edges.

There exist different noise types which can affect an image. The most well-known and studied noise types are the additive noise, the multiplicative noise and the impulse noise. The additive noise is based on adding to each pixel value a random value from a certain distribution, usually a Gaussian distribution leading to the Gaussian noise. On the other hand, the images corrupted with multiplicative noise are characterized by the fact that the noise depends on the intensity of the pixel. For instance, speckle noise is an example of multiplicative noise. Finally, the impulse noise is based on the replace of a percentage of the pixels by a fixed value (usually the minimum or the maximum possible value) or a random value (usually from a uniform distribution). The removal of this last noise type is going to be the main target of our contribution.

Impulse noise removal has been a recurring topic in these last years. Several methods have been proposed to remove this noise type. In [2] a decisionbased, signal adaptive median filtering algorithm for removal of impulse noise is proposed. In [3], a variation of the Windyga's peak-and-valley filter ([4]) based on a recursive minimum-maximum method, which replaces the noisy pixel with a value based on neighbourhood information is presented. In the paper [5], a new decision-based algorithm is proposed for restoration of images that are highly corrupted by impulse noise. The algorithm removes only corrupted pixels taking into account the median value of the values of its neighbouring pixel values. Then, in [6] a new impulse noise detector is presented. This detector is based on the differences between the current pixel and its neighbours aligned with four main directions. Next, the detector is combined with the weighted median filter to get a new directional weighted median filter. Finally, an impulse noise detector using mathematical residues is proposed in [1]. This method tries to identify pixels that are contaminated by the salt or pepper noise. The image is then restored using a sequence of open-close algorithms that it is applied only to the noisy pixels.

Beyond all the previous methods, some recent methods based on fuzzy logic have been proposed. For example, in [7] a new framework for reducing impulse noise from digital color images is presented, in which a fuzzy detection phase is followed by an iterative fuzzy filtering technique. In [8] a novel impulsive noise elimination filter combining a double noise detector with an adaptive neural fuzzy inference system is proposed. In addition, we can find methods to remove random impulse noise in color video sequences in the literature. For example, in [9] where different successive filtering steps are applied.

On the other hand, fuzzy mathematical morphology, which is the generalization of binary morphology [10] using techniques of fuzzy sets (see $[11,12,13,14])$, has shown a great potential in image processing. In particular, fuzzy mathematical 
morphology plays an important role in many applications like among others segmentation and edge detection (see $[15,16,17])$ and filtering (see $[18,19]$ ). Thus, in this paper we want to propose a novel filtering method for impulse noise removal by extending the method presented in [1] to fuzzy mathematical morphology. In this extension, the fuzzy mathematical operators and their fuzzy mathematical residues will be used in order to allow a better treatment and a representation with greater flexibility of the uncertainty and ambiguity present in any level of an image. In addition, this new method will show a better behaviour of the performance of the algorithm with respect to an increase of the amount of noise compared with the algorithm presented in [1]. The results are objectively compared using the wellknown Peak to Signal-Noise Ratio (PSNR) measure. Our method outperforms the non-fuzzy one for low amounts of noise while its performance is similar for images corrupted with high amounts of noise. Other non-linear filtering algorithms are also compared such as the standard median filter, an adaptive median filter and the Decision-Based algorithm proposed in [5].

The communication is organized as follows. In the next section, the impulse noise model and the definitions and properties of the fuzzy morphological operators are recalled. In Section 3 the proposed novel algorithm based on fuzzy mathematical morphology is explained. Then, in Section 4, the objective performance comparison based on PSNR among our method and some noise filtering algorithms is performed. Finally, in the last section, some conclusions and future work are pointed out.

\section{Basic definitions}

\subsection{Impulse noise models}

Images are often corrupted by impulse noise due to errors by noisy sensors or transmissions channels. Impulse noise refers to a wide variety of processes that result in the same basic image degradation: only a few pixels are corrupted, but they are very noisy. The effect is similar to sprinkling white and black dots (salt and pepper) on the image. One example is the "salt and pepper" noise which arises when transmitting images over noisy digital channels.

To be more precise, let $x_{i, j}$ be the gray level image of the original image $\mathbf{x}$ at the pixel location $(i, j)$. Denote by $\mathbf{y}$ a noisy image, then the observed gray level at the pixel location $(i, j)$ is given by

$$
y_{i, j}= \begin{cases}x_{i, j} & \text { with probability } 1-p \\ \nu_{i, j} & \text { with probability } p\end{cases}
$$

where $(i, j) \in\{1, \ldots, M\} \times\{1, \ldots, N\}$, and $\nu_{i, j}$ is an identically distributed, independent random process with and arbitrary underlying probability density function [20], that is the intensity value of the noisy pixel. There are two types of impulse noise, one is the "salt and pepper" noise and the other is random-valued impulse noise. In early impulse noise models, noisy pixels are often replaced by alternative values $s_{\min }$ and $s_{\max }$, where $\left[s_{\min }, s_{\max }\right.$ ] is the image dynamic range, so that, the observed gray level is given by

$$
y_{i, j}= \begin{cases}s_{\min } & \text { with probability } p \\ s_{\max } & \text { with probability } q \\ x_{i, j} & \text { with probability } 1-(p+q),\end{cases}
$$

where $r=p+q$ defines the noise level. The model (1) is a more general model in which the noisy pixel is taken as an arbitrary value in the dynamic range.

\subsection{Fuzzy logic morphological operators}

Fuzzy morphological operators are defined using fuzzy operators such as fuzzy conjunctions, like tnorms, and fuzzy implications. More details on these logical connectives can be found in [21] and [22], respectively.

Definition $1 A$ t-norm is a commutative, associative, non-decreasing function $T:[0,1]^{2} \rightarrow[0,1]$ with neutral element 1 , i.e., $T(1, x)=x$ for all $x \in[0,1]$.

Next we recall the definition of fuzzy implications.

Definition 2 A binary operator $I:[0,1]^{2} \rightarrow[0,1]$ is a fuzzy implication if it is non-increasing in the first variable, non-decreasing in the second one and it satisfies $I(0,0)=I(1,1)=1$ and $I(1,0)=0$.

A well-known way to obtain fuzzy implications is the residuation method. Given a t-norm $T$ the binary operator

$$
I_{T}(x, y)=\sup \{z \in[0,1] \mid T(x, z) \leq y\}
$$

is a fuzzy implication called the residual implication or $R$-implication of $T$.

Using the previous operators, we can define the basic fuzzy morphological operators such as dilation and erosion. We will use the following notation: $T$ denotes a t-norm, $I$ a fuzzy implication, $A$ a greylevel image, and $B$ a grey-level structuring element.

Definition 3 ([12]) The fuzzy dilation $D_{T}(A, B)$ and the fuzzy erosion $E_{I}(A, B)$ of $A$ by $B$ are the grey-level images defined by

$$
\begin{aligned}
& D_{T}(A, B)(y)=\sup _{x} T(B(x-y), A(x)) \\
& E_{I}(A, B)(y)=\inf _{x} I(B(x-y), A(x)) .
\end{aligned}
$$

From the fuzzy erosion and the fuzzy dilation, the fuzzy opening and the fuzzy closing of a grey-level image $A$ by a structuring element $B$ can be defined as follows. 
Definition 4 ([12]) The fuzzy closing $C_{T, I}(A, B)$ and the fuzzy opening $O_{T, I}(A, B)$ of $A$ by $B$ are the grey-level images defined by

$$
\begin{aligned}
& C_{T, I}(A, B)(y)=E_{I}\left(D_{T}(A, B),-B\right)(y), \\
& O_{T, I}(A, B)(y)=D_{T}\left(E_{I}(A, B)-B\right)(y) .
\end{aligned}
$$

A more detailed account on these operators, its properties and applications can be found in $[12,14,17,23]$. In particular, when $I$ is the residual implication of $T$, most of the usual properties of a mathematical morphology hold.

\section{The proposed algorithm}

The proposed algorithm is based on an extension to fuzzy mathematical morphology of the algorithm presented in [1] which is a classical mathematical morphology based method. The method is divided in two main steps. The first one is a preliminary identification of corrupted pixels in an effort to avoid the processing of pixels which are not corrupted by impulse noise. In the second one the filtering method is applied only to those pixels identified as noise in the first step.

\subsection{Fuzzy morphological residue detector}

The fuzzy mathematical morphological residues of Top-Hat and Dual Top-Hat operators are used to detect noisy pixels. Since the fuzzy opening removes salt impulse noise and the fuzzy closing removes pepper impulse noise, through the Top-Hat operators the salt and pepper pixels noises can be notably determined. In general, these transformations find structures which have been removed by the opening and closing filters and the residua between the original image and the filtered image increases notably the contrast of the erased regions (see [10]). So, the Top-Hat transformations are defined as follows

$$
\begin{aligned}
& \rho_{T, I_{T}}(A, B)=A \backslash O_{T, I_{T}}(A, B) \text { (Top-Hat) } \\
& \rho_{T, I_{T}}^{d}(A, B)=C_{T, I_{T}}(A, B) \backslash A \text { (Dual Top-Hat). }
\end{aligned}
$$

At this point, noise pixels are detected by comparing these two images with a threshold $T$ :

$$
r_{T, I_{T}}(A, B)= \begin{cases}1 & \text { if } \rho_{T, I_{T}}(A, B) \geq T \\ & \text { and } \rho^{d} I_{T, I_{T}}(A, B)=0 \\ -1 & \text { if } \rho_{T, I_{T}}(A, B)=0 \\ & \text { and } \rho^{d}{ }_{T, I_{T}}(A, B) \geq T \\ 0 & \text { otherwise. }\end{cases}
$$

Now, if $r_{T, I_{T}}(A, B)(i, j)=1$, then $A(i, j)$ is considered as salt noise or if $r_{T, I_{T}}(A, B)(i, j)=-1$, as pepper noise. When one of these two types of noisy pixels is detected, the corresponding generalized fuzzy open-close sequence of the next step is applied. If $r_{T, I_{T}}(A, B)(i, j)=0$, the pixel is uncorrupted and it remains without change.

\subsection{Fuzzy Open-Close Sequence Algorithm}

In this step, two filters using adequate fuzzy openclose sequences are applied to the corrupted pixels. The first one, which is called fuzzy open-close filter (FOCF) is defined as follows:

$$
F O C F_{T, I_{T}}\left(A,\left(B_{1}, B_{2}\right)\right)=C_{T, I_{T}}\left(O_{T, I_{T}}\left(A, B_{1}\right), B_{2}\right)
$$

where $B_{1}$ and $B_{2}$ are two structuring elements. This filter is applied to remove the salt noise pixels. In particular, the size of $B_{1}$ must be small enough to preserve the details of the image and the size of $B_{2}$ must be larger than of $B_{1}$ in order to eliminate powerfully the pepper noise pixels which have been not removed by the fuzzy opening.

In the same way, the fuzzy close-open sequence filter (FCOF) is defined as follows:

$\operatorname{FCOF}_{T, I_{T}}\left(A,\left(B_{1}, B_{2}\right)\right)=O_{T, I_{T}}\left(C_{T, I_{T}}\left(A, B_{1}\right), B_{2}\right)$

where $B_{1}$ and $B_{2}$ are again two structuring elements. Analogously to the previous filter, this filter is applied to remove the pepper noise pixels.

However, the noises whose size is larger than the size of $B_{1}$ will not be removed and in fact, they are propagated in the image. This fact leads to the generation of some undesired white (or black) blocks in the filtered image. To avoid this behaviour, the so-called Block Smart Erase (BSE) algorithm is applied. It is based on the median of the surrounding pixels. The details are the following:

1. Consider an $N \times N$ (it is recommended $N=5,7$ or 9) window centred at the test pixel.

2. If $A(i, j) \in\{0,255\}$ then we have an absolute extreme value and step 3 must be applied. Otherwise, the pixel is not altered.

3 . If an extreme value is detected, assign the median value of the window as its gray-level value.

Finally, the FMMOCS filter can be defined as the arithmetic mean of the two previous fuzzy open-close and close-open sequence filters after applying the BSE algorithm, that is, $F M M O C S_{T, I_{T}}\left(A,\left(B_{1}, B_{2}\right)\right)=$

$$
\begin{aligned}
& =\frac{B S E\left(F O C F_{T, I_{T}}\left(A,\left(B_{1}, B_{2}\right)\right)\right)}{2} \\
& +\frac{B S E\left(F C O F_{T, I_{T}}\left(A,\left(B_{1}, B_{2}\right)\right)\right)}{}
\end{aligned}
$$

\section{Simulation results}

In this section the performance of the proposed method will be evaluated and compared with other well-known methods for filtering noisy images which are corrupted by impulse noise. Comparisons are made with other nonlinear filtering techniques, specifically, a standard $3 \times 3$ and $5 \times 5$ median filter (SMF), an adaptive median filter (AMF) with a maximun allowed size of the adaptive filter window $\left(\mathrm{S}_{\max }\right)$ of 9 and 17, the Decision-Based algorithm (DBA) a nonlinear filter designed by Srinivasan and Ebenezer (DBA) in [5] and finally, the 


\begin{tabular}{|c|c|c|c|c|c|c|c|c|c|}
\hline Noise & \multicolumn{9}{|c|}{ PSNR } \\
\cline { 2 - 10 } & DBA & SMF3 & SMF5 & AMF9 & AMF17 & FMMOCS & OCS & FMMOCSflat & OCSflat \\
\hline 10 & 30,2896 & 32,2722 & 29,2999 & 37,1820 & 37,1820 & 33,5973 & 29,7633 & 32,2427 & 31,6873 \\
\hline 20 & 29,7204 & 28,4986 & 28,2710 & 34,7001 & 34,7001 & 31,5054 & 31,0747 & 31,5612 & 31,1085 \\
\hline 30 & 29,0282 & 23,4083 & 26,0472 & 32,8516 & 32,8216 & 30,5352 & 31,1533 & 31,1954 & 29,6786 \\
\hline 40 & 28,0453 & 18,5460 & 25,5084 & 30,7554 & 30,7554 & 29,9621 & 30,4075 & 30,3865 & 28,3672 \\
\hline 50 & 26,8784 & 14,8950 & 22,4622 & 29,2463 & 29,2463 & 29,2843 & 29,3507 & 29,3425 & 27,3714 \\
\hline 60 & 25,1899 & 12,1165 & 18,4381 & 27,3981 & 27,4294 & 28,2932 & 28,4292 & 28,4284 & 26,5269 \\
\hline 70 & 23,7784 & 9,74390 & 13,8772 & 24,9704 & 25,7660 & 27,1356 & 27,4667 & 27,4667 & 25,7700 \\
\hline 80 & 21,5853 & 7,84570 & 10,1319 & 18,7670 & 23,7004 & 25,1708 & 26,0970 & 26,0963 & 24,8901 \\
\hline
\end{tabular}

Table 1: PSNR for various filters for Lenna image at different noise densities.

open-close sequence (OCS) algorithm done in [1] and based on classical grey-level mathematical morphology. The performance of the algorithm is tested with different gray scale images such us "lenna.tif" an image with homogeneous regions and representative for low detail images, "cameraman.tif" and "lacornou.tif" for medium detail images, and the image "walkbridge.tif" and "baboon.tif" with high activity. Their dynamic range is $[0,255]$. In the simulations, images are corrupted by impulse noise ("salt and pepper" noise), where 255 represents "salt" and 0 represents the "pepper" noise with equal probability. Also a wide range of noise levels varied from $10 \%$ to $80 \%$ with increments of $10 \%$ will be tested. We have not increased the percentage of impulse noise for above the $80 \%$ because the methods remove effectively the noise but the edges are not preserved, except the SMF which performs badly in both aspects.

In this stage of our study the restoration performance is quantitatively measured by peak signalto-noise ratio (PSNR) defined by

$$
P S N R=10 \log _{10}\left(\frac{R^{2}}{M S E}\right)
$$

where $R$ is the maximum fluctuation in the input image and $M S E$ is the mean-squared error computed using the following expression:

$$
M S E=\frac{\sum_{m, n}\left(I_{1}(m, n)-I_{2}(m, n)\right)^{2}}{M \times N}
$$

where $I_{1}$ is the original image, $I_{2}$ the restored image, and $M \times N$ is the size of the images. More measures can be used, as can be seen in our previous works on denoising using fuzzy mathematical morphology $[24,25,26,27]$. In [28] the authors used the PSNR and the correlation, Srinivasan and Ebenezer in [5] measure the performance of the algorithm using the PSNR and the "image enhancement factor" (IEF), and in $[29,30]$ together with the PSNR the performance of the proposed algorithms is measured using the "mean absolute error" (MAE). The structural similarity index measure (SSIM) is used also in [31].
Recall that the proposed algorithm (FMMOCS) depends on the threshold $T$ and the size of the structuring element sequence. The parameter $T$ is easy to set if we consider Figure 1 where we represent PSNR versus $T$ for the five images used in the experiments. It is observed that there is a minimal variation of PSNR in a wide range of $T$, so we set $T$ in 90 , but it could have been taken even 0 as in [1], and the results would have changed minimally. Following our previous works on noise reduction and [1] we have fixed two types of structuring elements sequence: a flat sequence of squares of sizes 5,3 and 7 , respectively, and a binary sequence of diamonds with the same sizes. These structuring elements with sizes 5,3 and 7 correspond to the structuring elements $B, B_{1}$ and $B_{2}$, respectively, of the algorithm explained in Section 3. When the flat squares are used, we will denote the algorithm by "FMMOCSflat" and simply by "FMMOCS" when binary diamonds are considered. The same notation is used for the OCS algorithm proposed in [1].

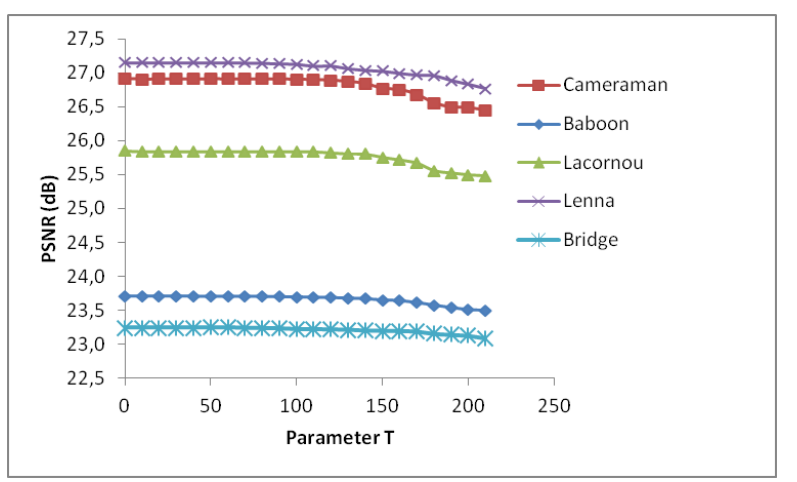

Figure 1: PSNR values for different values of $T$ for a $70 \%$ of noise density.

To quantitatively measure the performance of our filtering algorithm versus other methods, the results, measured with PSNR (dB), from $10 \%$ to $80 \%$ impulsive noise, are shown in Figure 2 (and table 1 for the Lenna image). It is seen that the performance of the proposed algorithm is better than or comparable to the other filters when the noise ratio is higher than $45 \%$ while it is only overcome 

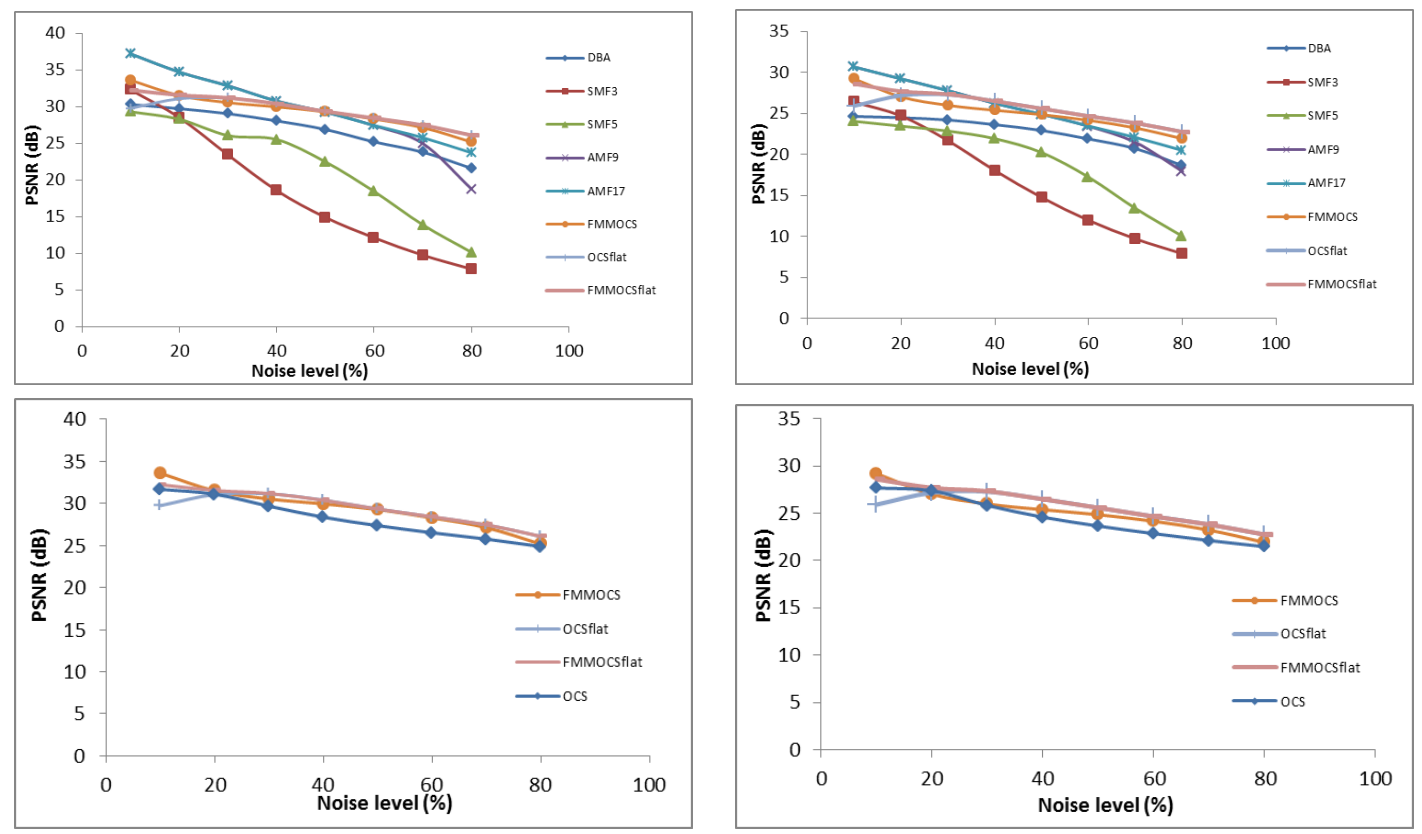

Figure 2: PSNR values for different filters operating on the images at various noise level. Left: "Lenna" image. Right: "walkbridge" image. Top: plots for all filters. Down: plot for open-close sequence based filters and the two types of structuring elements.

by SMF17 for lower amounts of noise. Also FMMOCS filter performs robustly over all the noise range and represents a slowly decreasing curve at the beginning of the range and when the noise ratio significantly increases. Other filters fall down abruptly generating worse results when the noise ratio is high. The performance of the DBA filter is similar to the proposed algorithm, but in our case the PSNR values are better. In the case of $90 \%$ all filters have a behaviour which a detail-preserving regularization is not obtained and the edges are not preserved. These conclusions can be extended to the other images since similar curves were obtained with these other ones. Also, in Figure 2 the plots of PSNR curves versus noise level for the "walkbridge" image can be observed. There, the odd behaviour of the OCS algorithm can be observed. Note that it obtains a worse result for the image corrupted with $10 \%$ of noise than for the one corrupted with $20 \%$. On the other hand, the FMMOCS is more robust in this sense since the curve is strictly decreasing as well as the rest of the algorithms. From Figure 3 to Figure 8 we present restoration results for several different noise levels and images. Note that in Figure 6 we display some of the restored images whose PSNR values were collected in Figure 2. As we can see from Figures 3, 7 and 8, the FMMOCS algorithm gives the best performance in terms of noise suppression and detail and edge preservation, at least for the noise levels shown. It is because the algorithm, from noise levels up to approximately $80 \%$, seems to locate the noise accurately. So, the proposed filter can remove most of the noise effectively while well preserving the edge image details of the image.

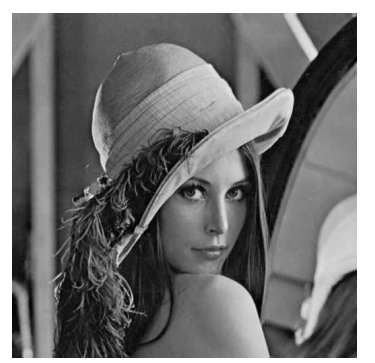

(a) Original image

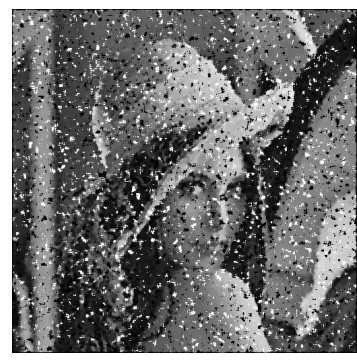

(c) SMF5

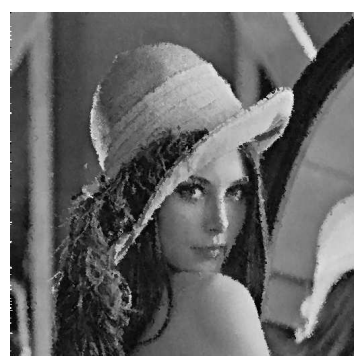

(e) DBA

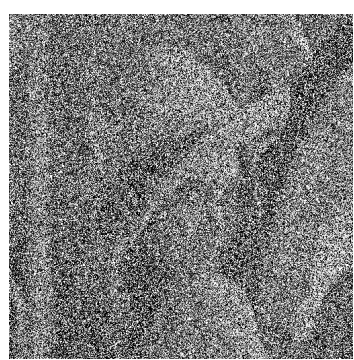

(b) Corrupted image

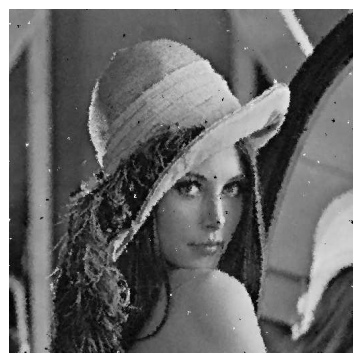

(d) AMF17

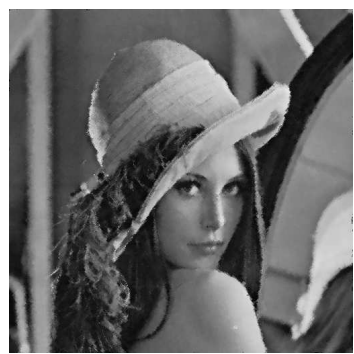

(f) FMMOCS
Figure 3: Simulation results of different filters for the Lenna image corrupted with $70 \%$ noise. See Table 1 for the PSNR values. 


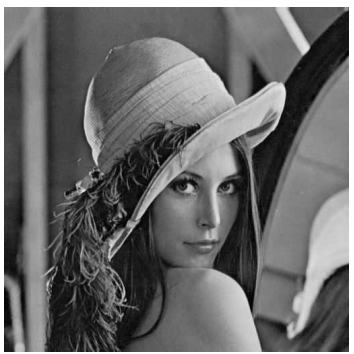

(a) Original image

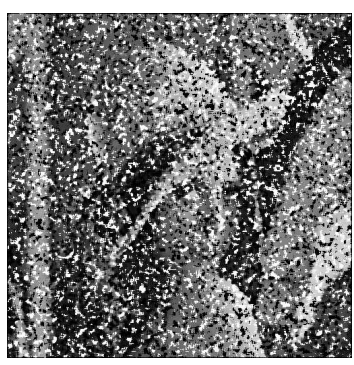

(c) SMF5

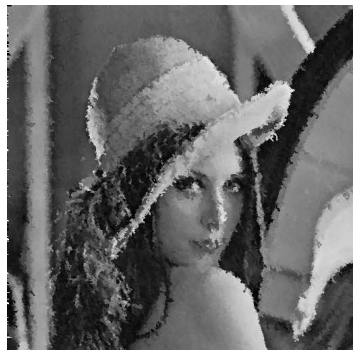

(e) DBA

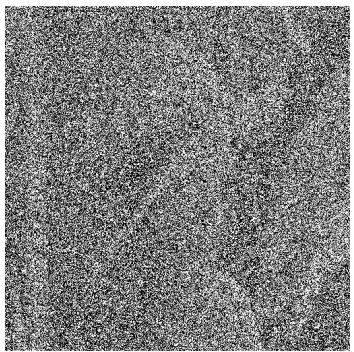

(b) Corrupted image

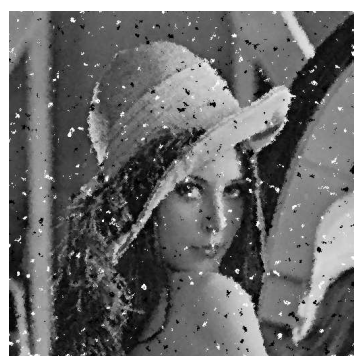

(d) AMF17

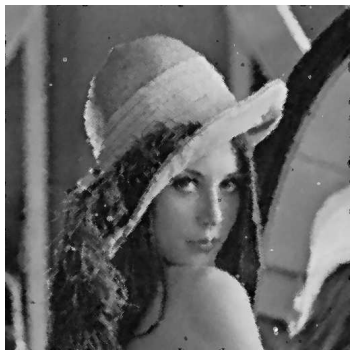

(f) FMMOCS
Figure 4: Simulation results of different filters for the Lenna image corrupted with $80 \%$ noise. See Table 1 for the PSNR values.

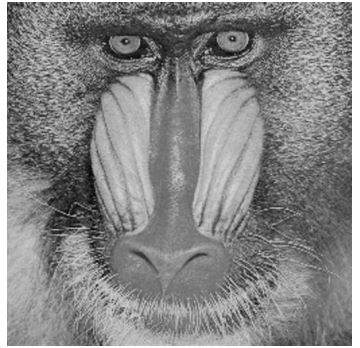

(a) Original image

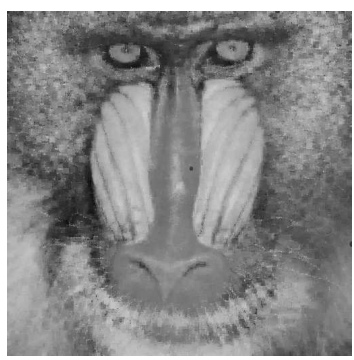

(c) FMMOCS

$(\mathrm{PSNR}=22.0782 \mathrm{~dB})$

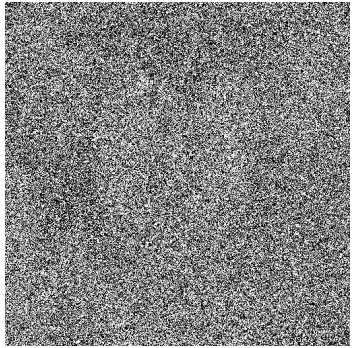

(b) Corrupted image

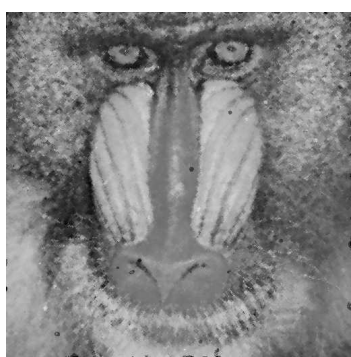

(d) FMMOCSflat

$(\mathrm{PSNR}=22.2992 \mathrm{~dB})$
Figure 5: Restoration results for FMMOCS filter for the "baboon" image with $80 \%$ noise using the two sequences of structuring elements.

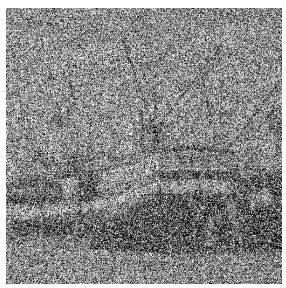
$60 \%$

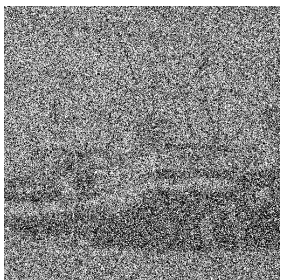

(c) Corrupted image $70 \%$

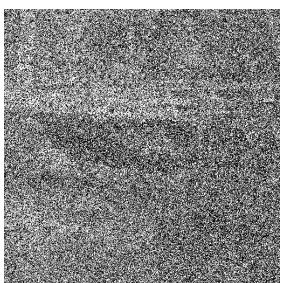

(e) Corrupted image $70 \%$

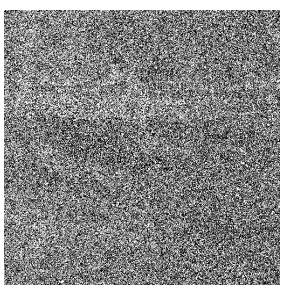

(g) Corrupted image $80 \%$ (a) Corrupted image

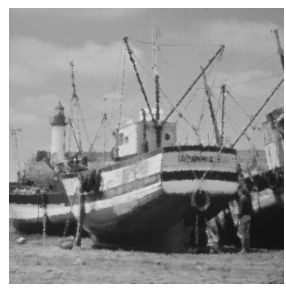

(b) FMMOCS $(\mathrm{PSNR}=26.8085 \mathrm{~dB})$

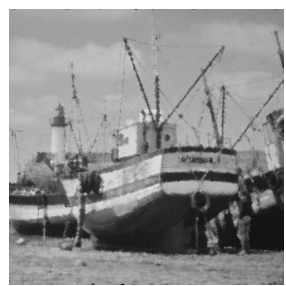

(d) FMMOCS $(\mathrm{PSNR}=25.8371 \mathrm{~dB})$

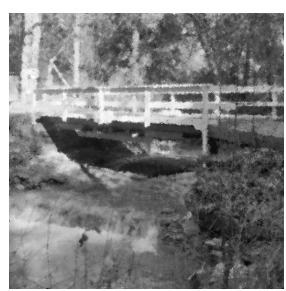

(f) FMMOCS $(\mathrm{PSNR}=23.2301 \mathrm{~dB})$

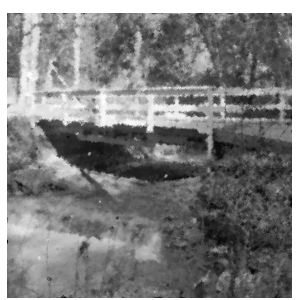

(h) FMMOCS

$(\mathrm{PSNR}=21.9147)$
Figure 6: Restoration results for FMMOCS using binary elements for the "walkbridge" and "lacornou images" corrupted with several amounts of noise.

\section{Conclusions and future work}

In this paper, we have presented a novel filtering method for impulse noise corrupted images based on the fuzzy mathematical morphology using t-norms. The algorithm extends the method presented in [1] to the fuzzy framework using the fuzzy mathematical operators and residues. The obtained results show that the new algorithm outperforms the nonfuzzy one and other well-established nonlinear filtering methods from both the visual point of view and the PSNR values. As future work, we want to deal with random values impulse noise since we hope that the fuzzy approach can be very competitive for its removal. In addition, the performance of the fuzzy mathematical morphologies based on uninorms could be worthy to study. 


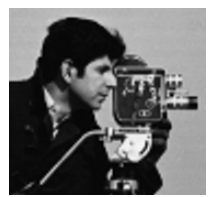

(a) Original detail

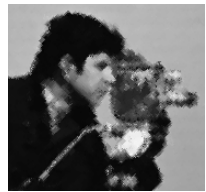

(d) FMMOCS

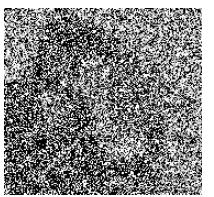

(b) Corrupted image

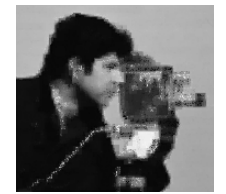

(e) OCSflat

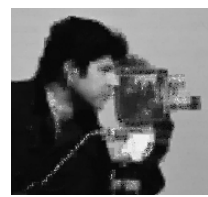

(c)

FMMOCSflat

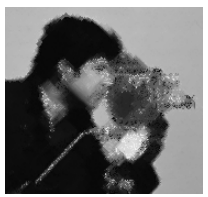

(f) OCS

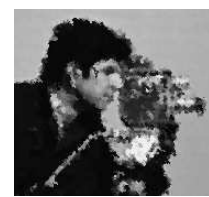

(g) DBA

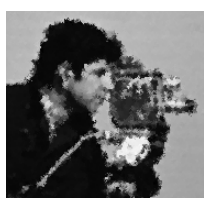

(h) AMF17
Figure 7: Detail of the "cameraman" image corrupted with $70 \%$ noise and its restoration results for several nonlinear filters.

\section{Acknowledgement}

Supported by the Government Spanish Grant MTM2009-10320, with FEDER support.

\section{References}

[1] D. Ze-Feng, Y. Zhou-Ping, and X. You-Lun. High probability impulse noise-removing algorithm based on mathematical morphology. IEEE Signal Processing Letters, 14(1):31-34, 2007.

[2] G. Pok, J.C. Liu, and A.S. Nair. Selective removal of impulse noise based on homogeneity level information. Trans. Img. Proc., 12(1):8592, 2003.

[3] N. Alajlan, M. Kamel, and E. Jernigan. Detail preserving impulsive noise removal. Signal Processing: Image Communication, 19(10):993 - 1003, 2004.

[4] P. S. Windyga. Fast impulsive noise removal. IEEE Trans. Image Proc., 10:173-179, 2001.

[5] K.S. Srinivasan and D. Ebenezer. A new fast and efficient decision-based algorithm for removal of high-density impulse noises. Signal Processing Letters, IEEE, 14(3):189-192, 2007.

[6] Y. Dong and S. Xu. A new directional weighted median filter for removal of random-valued impulse noise. IEEE Signal Processing Letters, 14(3):193-196, March.

[7] S. Schulte, V. De Witte, M. Nachtegael, D. Van der Weken, and E.E. Kerre. Fuzzy two-step filter for impulse noise reduction from color images. IEEE Transactions on Image Processing, 15(11):3567-3578, 2006.

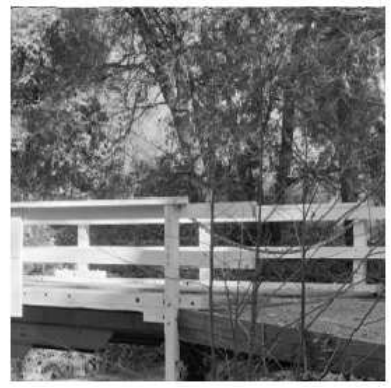

(a) Original detail

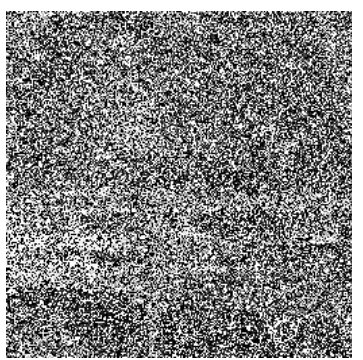

(b) Corrupted image $70 \%$

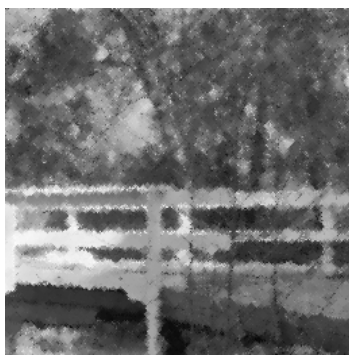

(d) FMMOCS

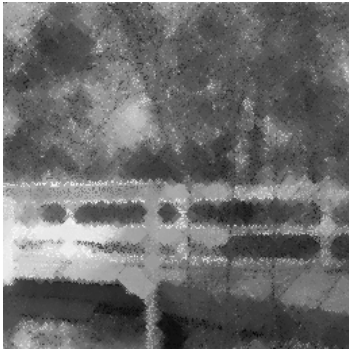

(f) $\mathrm{OCS}$

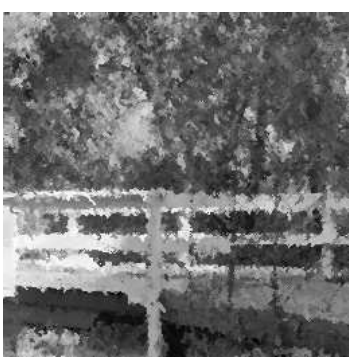

(h) DBA

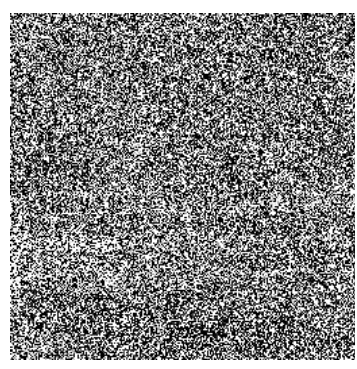

(c) Corrupted image $80 \%$

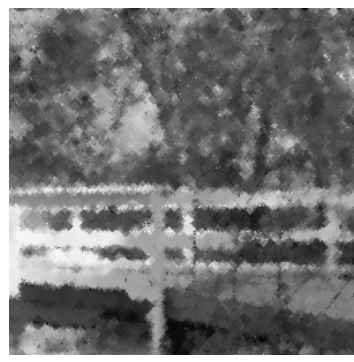

(e) FMMOCS

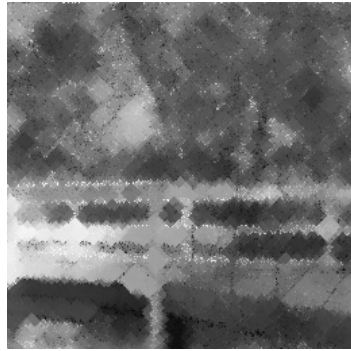

(g) OCS

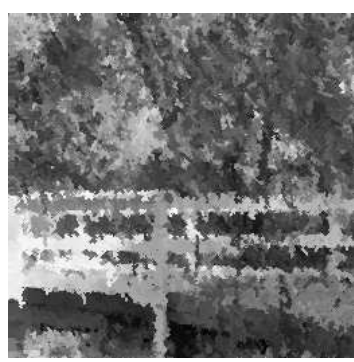

(i) DBA
Figure 8: Detail of the "walkbridge" image corrupted with two different amounts of noise and its restoration results for several nonlinear filters. See Figure 6 for the original image. 
[8] X. Wang, X. Zhao, F. Guo, and J. Ma. Impulsive noise detection by double noise detector and removal using adaptive neural-fuzzy inference system. Int. J. Electron. Commun., 65:429-434, 2011.

[9] T. Mélange, M. Nachtegael, and E.E. Kerre. Fuzzy random impulse noise removal from color image sequences. IEEE Transactions on Image Processing, 20(4):959-970, 2011.

[10] J. Serra. Image analysis and mathematical morphology, vols. 1, 2. Academic Press, London, 1982,1988.

[11] I. Bloch and H. Maître. Fuzzy mathematical morphologies: a comparative study. Pattern Recognition, 28:1341-1387, 1995.

[12] B. De Baets. Fuzzy morphology: A logical approach. In B. M. Ayyub and M. M. Gupta, editors, Uncertainty Analysis in Engineering and Science: Fuzzy Logic, Statistics, and Neural Network Approach, pages 53-68. Kluwer Academic Publishers, Norwell, 1997.

[13] B. De Baets, E.E. Kerre, and M. Gupta. The fundamentals of fuzzy mathematical morfologies part i: basics concepts. International J. of General Systems, 23:155-171, 1995.

[14] M. Nachtegael and E.E. Kerre. Fuzzy techniques in image processing, volume 52 of Studies in fuzziness and soft computing, chapter Classical and fuzzy approaches towards mathematical morphology, pages 3-57. SpringerVerlag, Heidelberg, 2000.

[15] Tung-Ching Su, Ming-Der Yang, TsungChiang Wu, and Ji-Yuan Lin. Morphological segmentation based on edge detection for sewer pipe defects on cctv images. Expert Systems with Applications, 38(10):13094 - 13114, 2011.

[16] Giuseppe Papari and Nicolai Petkov. Edge and line oriented contour detection: State of the art. Image and Vision Computing, 29(2-3):79 $-103,2011$.

[17] M. González-Hidalgo, A. Mir-Torres, D. RuizAguilera, and J. Torrens. Fuzzy morphology based on uninorms: Image edge-detection. opening and closing. In João Tavares and Natal Jorge, editors, Computational Vision and Medical Image Processing, pages 127-133, London, 2008. Taylor \& Francis Group.

[18] Romain Lerallut, Étienne Decencière, and Fernand Meyer. Image filtering using morphological amoebas. Image and Vision Computing, 25(4):395 - 404, 2007.

[19] Petros Maragos. Morphological filtering. In Al Bovik, editor, The Essential Guide to Image Processing (Second Edition), pages 293 321. Academic Press, Boston, second edition edition, 2009.

[20] S. Schulte, V. De Witte, Mike Nachtegael, D. Van der Weken, and E.E. Kerre. Fuzzy random impulse noise reduction method. Fuzzy Sets and Systems, 158(3):270 - 283, 2007.
[21] E.P. Klement, R. Mesiar, and E. Pap. Triangular norms. Kluwer Academic Publishers, London, 2000.

[22] M. Baczyński and B. Jayaram. Fuzzy Implications, volume 231 of Studies in Fuzziness and Soft Computing. Springer, Berlin Heidelberg, 2008.

[23] M. González-Hidalgo and S. Massanet. Closing and opening based on discrete t-norms. Applications to natural image analysis. In S. Galichet, J. Montero, and G. Mauris, editors, Proceedings of the 7th conference of the European Society for Fuzzy Logic and Technology (EUSFLAT-2011) and LFA-2011, volume 17 of Advances in Intelligent Systems Research, pages 358-365. Atlantis Press, 2011.

[24] M. González-Hidalgo, A. Mir-Torres, D. RuizAguilera, and J. Torrens. Applications of morphological operators based on uninorms. In ESTYLF-08, pages 203-210, Asturias, Spain, 2008. European Centre for Soft Computing.

[25] M. González-Hidalgo, A. Mir Torres, and J. Torrens Sastre. Noisy image edge detection using an uninorm fuzzy morphological gradient. Intelligent Systems Design and Applications, International Conference on (ISDA'09), pages 1335-1340, 2009.

[26] M. González-Hidalgo, A. Mir-Torres, and J. Torrens Sastre. Noise reduction using alternate filters generated by fuzzy mathematical operators using uninorms ( $\phi \mathrm{MM}-\mathrm{U}$ morphology). In P. Burillo, H. Bustince, B. De Baets, and J. Fodor, editors, EUROFUSE WORKSHOP 09, Preference Modelling and Decision Analysis, pages 233-238, Pamplona, Spain, 2009. Public University of Navarra.

[27] M. González-Hidalgo and S. Massanet. Closing and opening based on discrete t-norms. Applications to natural image analysis. In Proceedings of EUSFLAT-LFA 2011, pages $358-365$, Aix-Les-Bains, France, 2011.

[28] Hsiang-Chieh Chen and Wen-June Wang. Efficient impulse noise reduction via local directional gradients and fuzzy logic. Fuzzy Sets and Systems, 160(13):1841-1857, 2009.

[29] R.H. Chan, C.W. Ho, and M. Nikolova. Salt-and-pepper noise removal by median-type noise detectors and detail-preserving regularization. Image Processing, IEEE Transactions on, 14(10):1479-1485, 2005.

[30] T. Mélange, M. Nachtegael, S. Schulte, and E.E. Kerre. A fuzzy filter for the removal of random impulse noise in image sequences. Image and Vision Computing, 29(6):407 - 419, 2011.

[31] A. Jourabloo, A.H. Feghahati, and M. Jamzad. New algorithms for recovering highly corrupted images with impulse noise. Scientia Iranica, 19(6):1738 - 1745, 2012. 